\title{
ON THE APPLICATION OF LOAD RELAXATION IN CHARACTERIZING SUPERPLASTIC Al-Li 8090
}

\author{
F. Booeshaghi and H. Garmestani \\ FAMU-FSU College of Engineering, Department of Mechanical Engineering and Center for \\ Materials Research and Technology (MARTECH), Tallahassee, FL 32310-2175, USA
}

(Received September 22, 1997)

(Accepted in revised form August 25, 1998)

\begin{abstract}
$\underline{\text { Introduction }}$
High strain rate sensitivity is one of the main features of superplastic deformation which accounts for elongation to large strains $(\varepsilon \geq 1.5)(1,2,3)$. Strain-rate sensitivity is typically calculated as the slope of the $\log \sigma$ versus $\log \dot{\varepsilon}$ curve where $\sigma$ is the steady state flow stress and $\dot{\varepsilon}$ is the inelastic strain-rate. A variety of mechanical testing methods are used to evaluate strain-rate behavior including constant strain-rate, creep and other transient type testing methods such as strain-rate change test, and load relaxation tests. In this paper, the use of the load relaxation experiment to obtain the stress/strain rate behavior of an Al-Li 8090 superplastic material is investigated and compared to the strain-rate change tests. The strain rate change tests are found to be highly influenced by prior loading conditions and only valid for incrementally increasing tensile loads. Relaxation tests are found to be very effective for all ranges of strain rates. These tests can also be influenced by transient effects which are primarily due to anelasticity. Such transients can be avoided if the specimen is loaded to fully loaded (steady state) region prior to relaxation.
\end{abstract}

\section{Theoretical Background}

The majority of the testing methods used to measure strain rate sensitivity in superplastic materials rely on a sudden change in the applied strain-rate $(1,4,5)$. In such tests it is intended to calculate the strain-rate sensitivity at steady state for a large range of strain-rates. Strain-rate sensitivity parameter $m$, is defined as the slope of the log stress-log strain rate curve. Every point on the curve may represent a different test and sometimes performed on a completely different specimen. The calculation is based on the assumption that changes in structure and the resulting structural parameters are negligible. Such parameters can be temperature, strain and a set of internal state variables, $X$. (such as grain size, dislocation density, ... ) and temperature $\mathrm{T}$,

$$
m=\frac{\partial \log \sigma}{\partial \log \dot{\varepsilon}} \mid T, \varepsilon, X^{\prime} s
$$

The most widely used technique in the superplasticity community to measure strain rate sensitivity is the strain-rate change test $(1,2,4,6,7,8,9)$. It is used to explore the upper region of the strain-rate history (approximately greater than $10^{-5} \mathrm{~s}^{-1}$ ). The data from this test loses sensitivity at lower strain-rate 


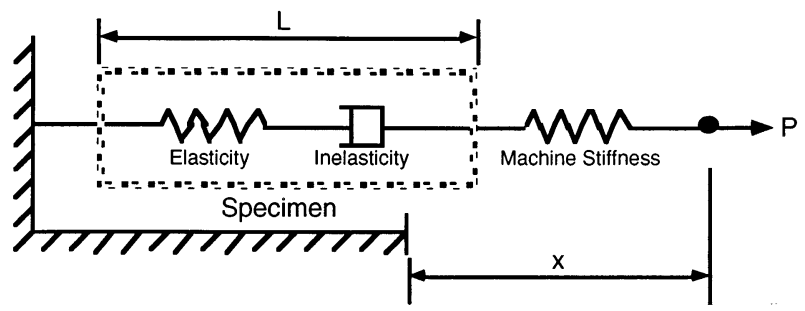

Figure 1. Schematic representation of the load relaxation test.

regimes due to machine limitations. The constant strain-rate test is also used $(10,11)$. The test provides large plastic deformations which result in strain hardening, and many tests are needed to characterize a single material. Strain hardening may be the reason for lack of popularity of the creep test in characterizing superplastic materials. In 1971, Lee and Hart (12) proposed that load relaxation was the proper test for characterizing strain-rate sensitive materials. This notion was later supported by others $(4,13,14,15)$. They showed that if load relaxation tests are performed under a precise control of the environment and the test temperature, a wider range of strain-rate histories could be explored. The derived data from these tests are highly affected by the presence of transients which reflect the non-steady state conditions. In the following the effect of such transients during load relaxation tests to measure strain rate sensitivity will be explained and compared to the more conventional tests of strain rate changes.

\section{Load Relaxation Test (LRT)}

During tensile deformation beyond elastic limit, the total strain rate $\dot{\varepsilon}_{\mathrm{ij}}$ is contributed by inelastic strain rate, $\dot{\varepsilon}_{\mathrm{ij}}^{\mathrm{i}}$, and elastic strain rate, $\dot{\varepsilon}_{\mathrm{ij}}^{\mathrm{e}}$.

$$
\dot{\varepsilon}_{\mathrm{ij}}=\dot{\varepsilon}_{\mathrm{ij}}^{\mathrm{e}}+\dot{\varepsilon}_{\mathrm{ij}}^{\mathrm{i}}
$$

In the load relaxation test, the material is deformed to a pre-determined strain (or extension) level and then the stroke or crossdead is held constant. During the load relaxation experiments, the total extension rate is forced to become zero, and what is measured is the rate of change of stress with time (Figure 1).

$$
\begin{gathered}
\dot{\varepsilon}_{\mathrm{ij}}^{\mathrm{i}}=-\mathrm{C} \dot{\sigma} \\
\mathrm{C}=\frac{\mathrm{A}_{0}}{\mathrm{~L}_{0}}\left(\mathrm{C}_{\mathrm{m}}+\frac{\mathrm{L}_{0}}{\mathrm{~A}_{0} \mathrm{E}}\right)
\end{gathered}
$$

where $\mathrm{C}$ is the combined elastic compliance, $C_{m}$ is the machine compliance, $L_{0}$ is the original specimen gage length, $A_{0}$ is the original specimen cross-sectional area, and $E$ is the specimen elastic modulus. The total unrecoverable plastic strain stored during the relaxation process is essentially a function of the elastic deformation of the material, the machine stiffness and the relaxation time (16), and is no more than $0.2 \%$ for most experiments. It can be concluded that the experimental result for such a test will then represent one constant structure or one state of hardness (17). Some of the other factors influencing the final results are the machine response time, temperature stability, and data sampling rate. Another crucial parameter in validating the flow stress response to changes in strain-rate is the machine response time. The environmental temperature stability and isolation from vibration (which may become substantial for hydraulic type testing machines) are among other important experimental variables. The 
flow stress of the material is directly related to the temperature of the material and must remain constant to correctly evaluate the material response. The temperature stability is specially important towards the latter part of the test when an accurate measure of lower strain rate regime are desired (13).

\section{Strain-Rate Change Test (SRCT)}

The strain-rate change test is typically conducted by deforming a material in uniaxial tension at a low strain-rate until steady state is reached and then increasing (or decreasing) the strain-rate instantly to a new level. The new strain-rate is then applied until the new steady state region is reached and the process is repeated, Figure 3. Each successive strain-rate is maintained until transient effects are diminished, or the steady state is reached. This can take up to $1 \%$ strain or greater at high temperatures. This means that a large amount of inelastic strain is stored during the total run. This is alarming since the main reason that the test is usually utilized is to find strain-rate effects at relatively constant strains (constant structure-hardness). Hence, the interpretation of the strain-rate change test results is typically based on the assumption that the strain hardening effects are negligible. At best one may assume that the plastic strain stored during the test process does not affect the results. Also, the correct determination of the time duration for the transient region is very important in the analysis of the SRCT data. The theoretical analysis of this effect requires proper modeling and is beyond the scope of this paper. Hence, in the analysis of the resultant data the increase in the contribution of the transient (anelasticity) effects must be considered. The test may be run in a decremental mode as well, but earlier studies have shown that the results may not be very reliable (4).

\section{Experimental Procedure and Material}

The tests were performed using a screw driven load frame equipped with a standard interchangeable load cell with an accuracy of $\pm 0.1 \%$ and a three-zone clamshell type resistance furnace with a sensitivity of $\pm 0.5^{\circ}$. The temperatures used were $400^{\circ} \mathrm{C}, 450^{\circ} \mathrm{C}$, and $516^{\circ} \mathrm{C}$. Strain-rates of $5 \times 10^{-4}$ $\mathrm{s}^{-1}, 5 \times 10^{-3} \mathrm{~s}^{-1}, 5 \times 10^{-2} \mathrm{~s}^{-1}$, and $5 \times 10^{-1} \mathrm{~s}^{-1}$ were applied. The sampling rate used here progresses from a high speed data acquisition of $40 \mathrm{~Hz}$ to $10 \mathrm{~Hz}$ and finally to $1 \mathrm{~Hz}$ so that enough data can be recorded for each decade of strain-rate. The external environmental temperature was stabilized by using a test chamber which enclosed the load frame and the accompanying components and maintained a uniform environmental temperature throughout the test.

The material used in this study was an 8090 Al-Li alloy of nominal composition Al-2.39 Li-1.21 $\mathrm{Cu}-0.64 \mathrm{Mg}-0.12$ (in wt\%). Test samples were cut out in a dog bone shape from rolled sheets of the material along the rolling direction. The dimensions of the sample were: gage length $=14.93 \mathrm{~mm}$, width $=2.83 \mathrm{~mm}$, and thickness $=2.46 \mathrm{~mm}$. Extreme caution was employed during machining to avoid introducing surface defects. Details of microstructural analysis and microtexture evolution of these specimens have been reported earlier $(18,19,20,21)$.

\section{$\underline{\text { Results and Discussion }}$}

In the following, strain-rate change test and load relaxation data for Al-Li 8090 at three temperatures $\left(516^{\circ} \mathrm{C}, 450^{\circ} \mathrm{C}\right.$, and $\left.400^{\circ} \mathrm{C}\right)$ are presented covering superplastic and non-superplastic behaviors. Using equations (3) and (4) and the load versus time data from load relaxation, the log stress-log strain rate curves were calculated and plotted in Figure 3. These curves covered over six decades of strain-rates. The relaxation data at various temperatures clearly identified region-II of superplastic deformation at a strain rate of $10^{-4} \mathrm{sec}^{-1}$. 


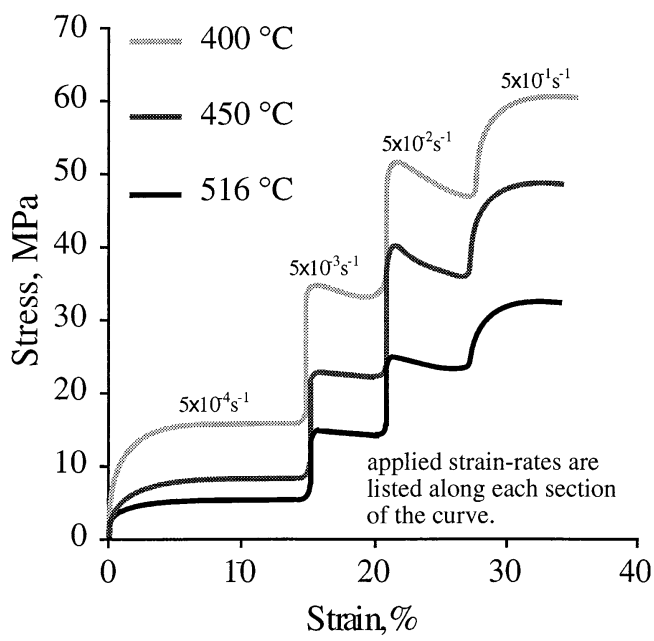

Figure 2. SRCT with incremental strain-rates.

Figure 2 represents the strain-rate change test data for three temperature levels and four applied strain-rates covering three decades of strain rates. The superposition of Figures 2 and 3 provided us with the Figure 4. It was clear that the stress relaxation results (below $5 \times 10^{-4} \mathrm{sec}^{-1}$ ) could be viewed as the lower strain-rate region of an overall test. Together with the strain-rate change test, they provided a master curve for the familiar S-shape log stress versus log strain-rate data which is usually observed in superplastic materials. The low strain-rate region of the state curve represented a different behavior is identified as the region 0 of $\log \sigma-\log \dot{\varepsilon}(13)$.

\section{Loading Transients and Steady State}

The result of the load relaxation experiments (as well as other strain rate change tests) are affected by transients due to the initial loading or reloading. The experimental results show the existence of

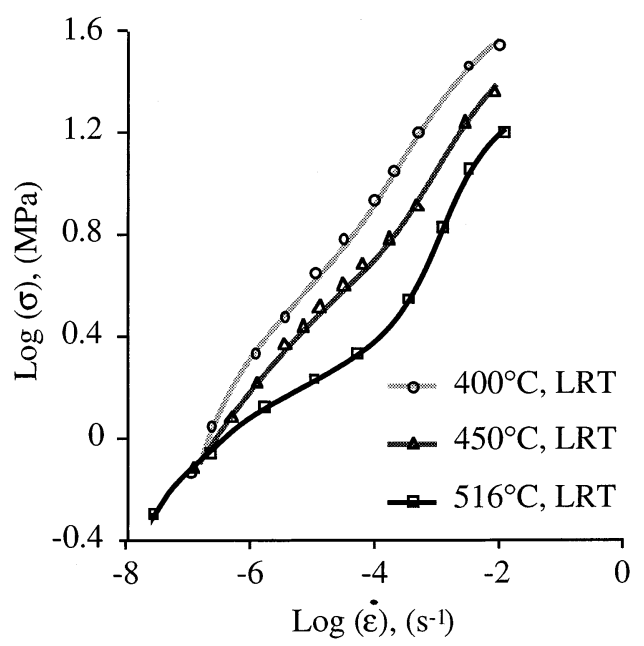

Figure 3. State curves for Al-Li 8090 from LRT. 


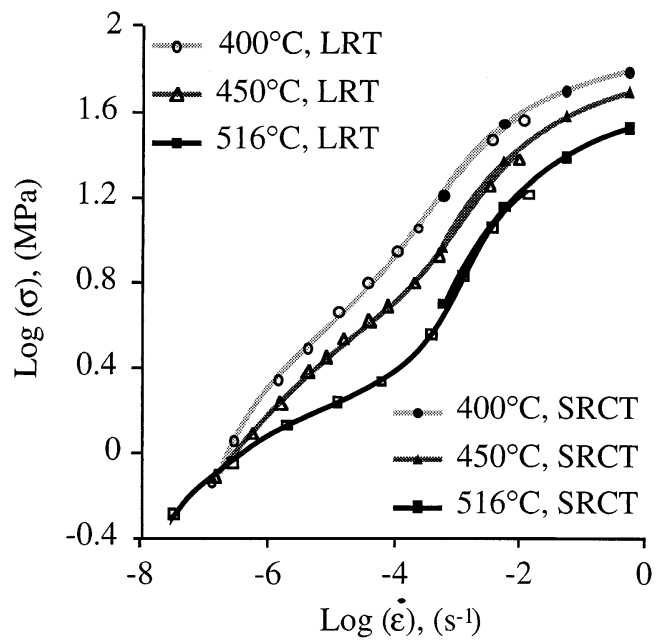

Figure 4. Composite test results for both the strain rate change test (SRCT) and the load relaxation test.

transient strain rate which is associated with the state of strain of the specimen beyond the homogeneous elastic strain $(22,23)$. That component of strain becomes zero when the state of strain is at equilibrium with the applied stress. The total inelastic strain rate of a specimen can be considered to be the sum of the anelastic strain rate $\dot{\varepsilon}_{\mathrm{ij}}^{\mathrm{a}}$, the strain rate due to grain boundary sliding $\dot{\varepsilon}_{\mathrm{ij}}^{\mathrm{gb}}$, and the contribution from the matrix plastic deformation $\dot{\alpha}_{\mathrm{ij}}$,

$$
\dot{\varepsilon}_{\mathrm{ij}}^{\mathrm{i}}=\dot{\varepsilon}_{\mathrm{ij}}^{\mathrm{a}}+\dot{\varepsilon}_{\mathrm{ij}}^{\mathrm{gb}}+\dot{\alpha}_{\mathrm{ij}}
$$

where $\dot{\varepsilon}_{\mathrm{ij}}^{\mathrm{a}}$ is the anelastic strain rate, $\dot{\varepsilon}_{\mathrm{ij}}^{\mathrm{gb}}$ is the grain boundary sliding, and $\dot{\alpha}_{\mathrm{ij}}$ is the contribution from the matrix plastic deformation. The main characteristics of the anelastic component are that it obeys a constitutive law that is essentially linear, and that is recoverable (23). Anelastic strain is linearly proportional to an internal stress (anelastic stress), $\sigma_{\mathrm{ij}}^{\mathrm{a}}(24)$. For the present study the simple power law introduced by Hart (24) can be used for the inelastic strain rate

$$
\dot{\varepsilon}_{\mathrm{ij}}^{\mathrm{a}}=\mathrm{L}\left(\sigma_{\mathrm{ij}}-\mu \varepsilon_{\mathrm{ij}}^{\mathrm{f}}\right)
$$

where $\mu$ is defined as the anelastic modulus or the relaxation constant and $\sigma_{\mathrm{ij}}$ is the applied stress. A more complicated form of equation 6 can also be presented and used (25). The grain boundary sliding portion also satisfies a highly non-Newtonian viscous flow law (17). This behavior was deduced to arise from a combined effect of an intrinsic Newtonian sliding friction for the grain boundary and the grain matrix plastic flow at grain corners that is required for compatibility (13).

The effect of the anelastic component is prominent only during abrupt changes of the applied stress. This occurs principally upon initial loading and reloading or load reversals. The specimen was loaded at a temperature of $516^{\circ} \mathrm{C}$ and an applied strain-rate of $5 \times 10^{-3} \mathrm{~s}^{-1}$ for $20 \%$ followed by an abrupt change of strain-rate to $5 \times 10^{-4} \mathrm{~s}^{-1}$ until failure (Figure 5). The resulting stress strain curve was compared with constant strain-rate tests data at strain rates of $5 \times 10^{-4} \mathrm{~s}^{-1}$ and $5 \times 10^{-3} \mathrm{~s}^{-1}$. It was observed that with decremental SRCT the abrupt decrease in strain-rate caused the flow stress to decrease sharply, and approximately $10 \%$ strain was needed for the flow stress to climb back to the steady state level. Hence, the decremental SRCT requires a strain proportional to the change in the stress and the final strain rate for each change in strain-rate to reach steady state flow stress (in this case a strain of 10\%). If it is intended to isolate the combined effect of the grain boundary sliding and matrix 


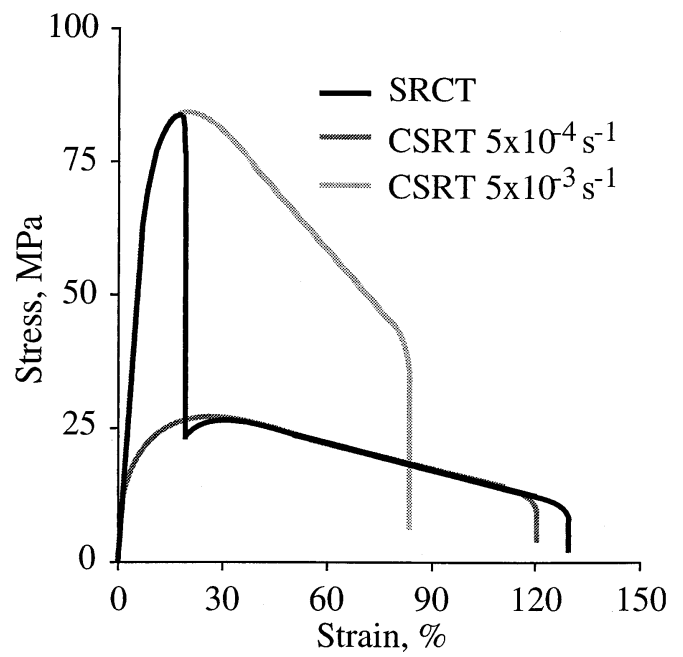

Figure 5. SRCT with decremental strain-rates.

deformation, it is necessary to eliminate the transients due to anelasticity. This can be performed by initiating the test in the fully loaded region (steady state) or subtract the anelastic strain rate from the calculated strain rate.

The steady state can now be defined as the condition for which for a given applied strain rate, the rate of change of the anelastic strain becomes negligible compared to the inelastic strain rate.

$$
\frac{\mathrm{d} \varepsilon_{\mathrm{ij}}^{\mathrm{a}}}{\mathrm{dt}} \cong 0, \text { or } \frac{\mathrm{d} \sigma_{\mathrm{ij}}^{\mathrm{a}}}{\mathrm{dt}} \cong 0
$$

If we assume a power law relationship for the combined effect of the plastic strain rate and the grain boundary sliding and if the load relaxation is initiated from the non-steady state condition, the stress history will follow a transient path as shown in Figure 6. A log stress-log strain rate plot may be shifted

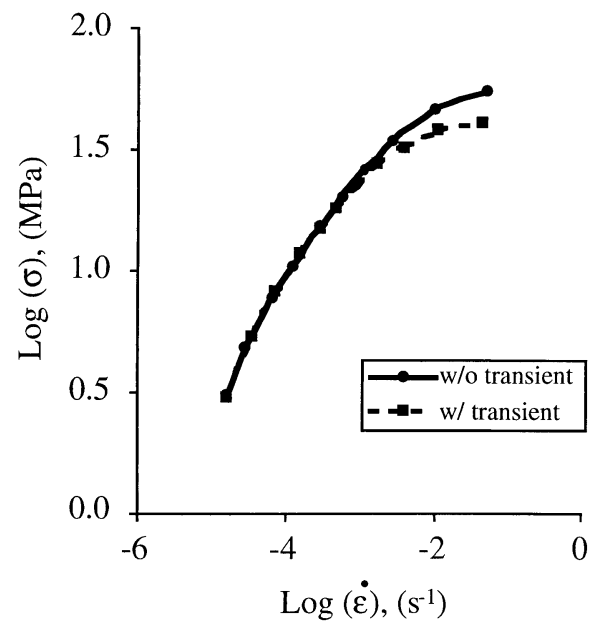

Figure 6. The $\log \sigma-\log \dot{\varepsilon}$ for Al-Li 8090 at $371^{\circ} \mathrm{C}$. 


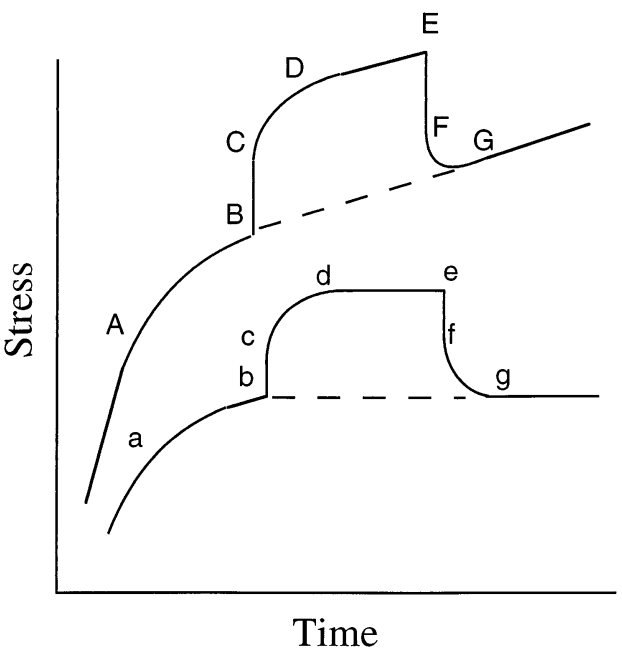

Figure 7. Schematic diagram for strain-rate change test strain rate data for a power law type material.

to the right or the higher strain rate regime according to equation 5 if the anelastic strain rate is not zero. The relaxation curve would finally join the main curve at a lower strain rate and at steady state. The transition from elasticity to steady state is usually very gradual and follows a nonlinear behavior (a to $\mathrm{b}$ or $\mathrm{A}$ to $\mathrm{B}$ in Figure 7). The region in which this transition occurs is defined as the transient region.

Reloading or a change in the strain rate from the steady state (points B, or b in Figure 7) will result in a new transient region to the new steady state $(\mathrm{D}$, or $\mathrm{d})$. The new steady state is related to the condition that $\dot{\varepsilon}_{2}=0$. During this transition, the apparent inelastic strain rate will be lower than the steady state stress $\left(\sigma^{\mathrm{ss}}\right)$. The time (or strain) required to reach such a condition is of course dependent on the difference from the steady state stress and the initial stress, $\sigma_{i}$, or the stress before the jump

$$
\Delta \varepsilon=\frac{\sigma_{\mathrm{ss}}-\sigma_{\mathrm{i}}}{\mu}
$$

Using this equation, the anelastic modulus, $\mu$, can be calculated to be $550 \mathrm{MPa}$ for the superplastic temperature $\left(516^{\circ} \mathrm{C}\right)$. The time required to reach such a condition is of course dependent on the final strain rate $\Delta \mathrm{t}=\Delta \varepsilon / \dot{\varepsilon}$. Very short times are necessary in the transition to high strain rates and much longer times are needed in the transition to lower strain rates. This increased time may not change the nature of the anelasticity but will contribute to more plastic deformation due to matrix and grain boundary sliding. This reduces the effectiveness of the strain rate tests in the case of transitions to the lower strain rates. One primary factor behind such tests for superplastic materials is the dominance of grain boundary sliding at a specific strain rate and temperature. Below and above superplastic strain rates the contribution from grain boundary sliding will reduce and other matrix dominated phenomena (work hardening, increase in grain size) may become activated.

\section{Conclusion}

The effect of transients during load relaxation tests have been investigated for superplastic Al-Li 8090 . The load relaxation test provided strain rate sensitivity for a large range of strain-rates with negligible plastic strain. The strain-rate change test provided high strain-rate data, but the low strain-rate portion of the method may be affected by transients and strain hardening. Load relaxation test was a very 
effective technique to measure strain-rate sensitivity for strain-rates lower than approximately $5 \times 10^{-3}$ $\mathrm{s}^{-1}$, as the strain-rate change test was used for strain-rates above this value. It is also shown that if the load relaxation tests is initiated at steady state condition (free of transients) the result of the load relaxation test can provide the low strain rate regime of the stress/strain rate curve commonly obtained using creep tests.

\section{Acknowledgments}

This research was supported by a NASA Grant NCC-1252, through Cennas-FAMU and the Center for Materials Research and Technology at Florida State University.

\section{References}

1. A. K. Ghosh and C. H. Hamilton, Metallurgical Trans. A. 10A, 699-706 (1979).

2. R. E. Goforth and M. N. Srinivasan, J. Testing Eval. JTEVA. 21, 36-43 (1993).

3. C. H. Hamilton, C. C. Bampton, and N. F. Paton, in Superplasticity in High Strength Aluminum Alloys, ed. N. E. Paton and C. H. Hamilton, TMS/AIME, Warrendale, PA (1982).

4. J. Hedworth and M. J. Stowell, J. Mater. Sci. 1061-1069 (1971).

5. T. G. Langdon and P. Yavari, Scripta Metall. 17, 435-440 (1983).

6. A. Arieli and A. Rosen, Metall. Trans. A. 8A, 1591-1596 (1977).

7. N. Furushiro, M. Toyoda, and S. Hori, Acta Metall. 36, 523-529 (1988).

8. H. Yoshinga, Z. Horita, and H. Kurishita, Scripta Metall. 29, 1815-1824 (1981).

9. A. Aran, Scripta Metall. 13, 843-846 (1979).

10. M. T. Cope, D. R. Evetts, and N. Ridley, J. Mater. Sci. 21, 4003-4008 (1986).

11. A. Wisbey, P. G. Partridge, and A. W. Bowen, J. Mater. Sci. 27, 3925-3931 (1992).

12. D. Lee and E. W. Hart, Metall. Trans. 2, 1245-1248 (1971).

13. F. Booeshaghi and H. Garmestani, Scripta Mater. 38, 89-94 (1998).

14. F. U. Enikeev and M. I. Mazurski, Scripta Metall. Mater. 32, 1-6 (1995).

15. T. K. Ha and Y. W. Chang, Scripta Metall. Mater. 32, 809-814 (1995).

16. E. W. Hart and H. Garmestani, J. Exp. Mech. 1-6 (1993).

17. E. W. Hart, Acta Metall. 15, 1545-1549 (1967).

18. F. Booeshaghi, H. Garmestani, and P. N. Kalu, Phenomenological Modeling of Superplasticity, 126th TMS Annual Meeting, Orlando, FL (1997).

19. H. Garmestani, P. Kalu, and D. Dingley, Microcharacterization of Al-8090 Superplastic Materials Using Orientation Imaging Microscopy, ed. L. S. A. o. T. M. Society (1995).

20. H. Garmestani, P. N. Kalu, and F. Booeshaghi, Mater. Sci. Forum, 243-245, 569-574 (1997).

21. H. Garmestani, P. Kalu, and D. Dingley, J. Mater. Sci. Eng. A. 242/1-2, 284-291 (1998).

22. E. W. Hart, J. Eng. Mater. Technol. 98, 193-202 (1976).

23. C. Zener, Elasticity and Anelasticity of Metals, The University of Chicago Press, Chicago (1948).

24. E. W. Hart, J. Eng. Mater. Technol. 106, 322-325 (1984).

25. E. W. H. Wei-Yan Shih, A Ci/limb Limited Theory for Metal Creep, The Seventh National Conference on Theoretical and Applied Mechanics, Taipei, Taiwan (1993). 